\title{
Métodos para documentar y restaurar zonas de difícil acceso en el Castillo de Salobreña. (Granada)
} Antonio Almagro Gorbea ${ }^{a}$, Antonio Orihuela Uzal ${ }^{\text {b }}$

Escuela de Estudios Árabes, CSIC, Granada, España, ${ }^{a}$ aalmagro@eea.csic.es , borihuela@eea.csic.es

\begin{abstract}
Construction fortifications sometimes are in places located in high mountains where it is very difficult to access them to obtain information about the restoring.

In The Salobreña Castle, Andalusí Fortification in the coast of Granada and remodeled at the end of the $\mathrm{XV}$ century, exists walls and fortifications located at the top of high cliff, therefore it is very difficult to obtain information using classic photogrammetric methods. The same case in restoration because it is difficult too.

A little multicopter has been used to solve the documentation problem and a compact camera was fixed in the multicopter. It is used to take a lot of pictures, obtaining such stereoscopic pairs as groups of pictures to modify with a program of photogrammetric scan.

A skilled worker has restored the elements located in high cliff using climbing equipment
\end{abstract}

Keywords: multicóptero, fotogrametría, trabajos de altura.

\section{Introducción}

Con notable frecuencia, los castillos y otras obras de fortificación ocupan lugares agrestes y de difícil acceso buscando las mayores dificultades para los potenciales atacantes. Esto suele generar problemas, tanto en la documentación de los mismos como en las posteriores intervenciones de restauración, que en tales casos requieren de costosos medios auxiliares para efectuar reparaciones que, en ocasiones, pese a ser imprescindibles resultan de costo insignificante frente al de los medios auxiliares necesarios para realizarlos, lo que muchas veces provoca que no se lleguen a ejecutar. En la toma de datos, las disponibilidades de medios auxiliares suelen ser siempre muy limitadas por lo que estos impedimentos generan dificultades muy serias.

Nuevos instrumentos que la tecnología va poniendo a nuestra disposición permiten resolver las dificultades de documentación, generalmente aprovechando las grandes ventajas que la foto- grametría digital ofrece. Pero también están resultando de gran utilidad los nuevos medios disponibles para obtener las fotos. Por otro lado, determinadas sistemas, no convencionales, de ejecutar las obras resuelven y economizan su realización.

El castillo de Salobreña es una fortificación de época andalusí situado en la costa granadina. Se asienta en lo más alto de la población sobre la cúspide de un cerro rocoso, casi aislado, situado en la llanura aluvial que se forma en la desembocadura del río Guadalfeo.

El cerro, que en tiempos pasados estuvo en el mismo borde del litoral, hoy se encuentra alejado del mismo más de quinientos metros por efecto de los aluviones que ha ido depositando el río. Mientras que por el este y el norte las pendientes han permitido desarrollarse a la población, al sur y al oeste la presencia de grandes 
acantilados sirvió de base a la construcción de la fortaleza cuyas murallas se asoman a sus mismos bordes. Estos acantilados superan en ocasiones los treinta metros de altura presentando en muchos lugares incluso pronunciados extraplomos.

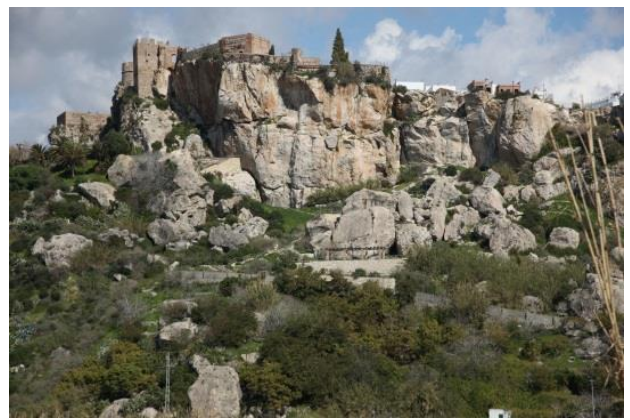

Fig. 1- Acantilado del lado sur del Castillo de Salobreña.

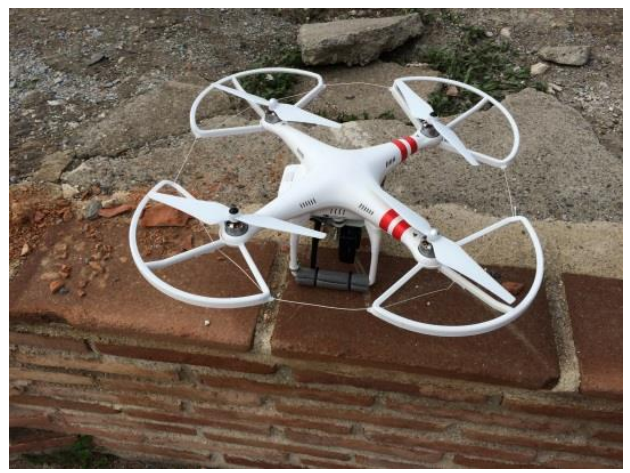

Fig. 2- Cuadricóptero con cámara adaptada para la toma de fotografías.

Estas circunstancias hacen muy difícil la documentación por métodos fotogramétricos clásicos, ya que las distancias a las que pueden tomarse las fotografías son excesivas y si nos aproximamos tendríamos un ángulo de inclinación muy grande y con zonas ocultas. Tampoco el uso de un escáner de láser, por la gran distancia a la que se tendrían que situar los instrumentos de toma de datos, permite una adecuada documentación de estas estructuras. La restauración de los paramentos de la muralla y sus baluartes en esas zonas resulta así mismo de gran dificultad y coste, si se deben hacer usando andamios convencionales.

\section{Documentación de sitios inaccesibles}

Para resolver el problema de la documentación de las murallas situadas en posiciones elevadas y muy distantes de los sitios accesibles para poder tomar las fotografías, se ha recurrido a un pequeño multicóptero al que se ha fijado una cámara fotográfica compacta Ricoh GRD. Esta cámara, de distancia focal fija y que permite enfoque manual, es la misma utilizada en algunos de nuestros levantamientos fotogramétricos y está calibrada para su uso en estereofotogrametría. Al multicóptero se le ha acoplado una pequeña cámara de video con su correspondiente emisor que permite controlar lo que está captando la cámara fotográfica, pudiendo de este modo dirigir el ingenio y obtener un recubrimiento fotográfico óptimo. La cámara permite hacer tomas de manera secuencial mientras está volando. Luego se seleccionan las fotos más adecuadas para su uso con los distintos programas.

La cámara puede montarse lo mismo con el eje óptico horizontal, para documentar alzados, como vertical, para tomas que permitan la restitución de plantas y elementos horizontales.

En el castillo de Salobreña hemos aplicado este sistema a la documentación del baluarte artillero ubicado en el extremo sur del castillo, que presenta su frente principal, que mira al mar, justo en el borde del acantilado, que en esa zona tiene más de veinte metros de altura. Con los medios ya descritos se ha podido hacer un barrido fotográfico, tanto de fotos horizontales como verticales, obteniendo pares estereoscópicos y bloques de fotografías para su tratamiento mediante el programa de escaneado fotogramétrico PhotoScan. Por este medio se ha conseguido un modelo 3D y, a partir del mismo, las correspondientes ortofotos de la planta y de los alzados de la fortificación. Para la orientación y escalado del modelo contábamos previamente con puntos de control medidos al realizar el levantamiento taquimétrico de todo el conjunto.

La toma de datos fue realizada en un tiempo muy breve, de apenas pocos minutos, la mayor parte de los cuales se invirtieron en la preparación del disposiitivo para el vuelo. El trabajo de gabinete requirió unas pocas horas hasta la obtención de las ortofotos, que constituyen de 
por si documentos de gran utilidad. Si se quieren obtener dibujos vectoriales, basta con insertar las ortofotos y darles escala en Auto Cad y dibujar sobre ellas las líneas que se consideren relevantes. Una combinación del dibujo vectorial sobrepuesto a la ortofoto puede resultar el documento más eficaz.

Este procedimiento ha resultado de gran versatilidad, rapidez y eficacia para la documentación de este tipo de situaciones, y pensamos que en el futuro se podrá utilizar ampliamente para resolver éstas y otras dificultades parecidas.

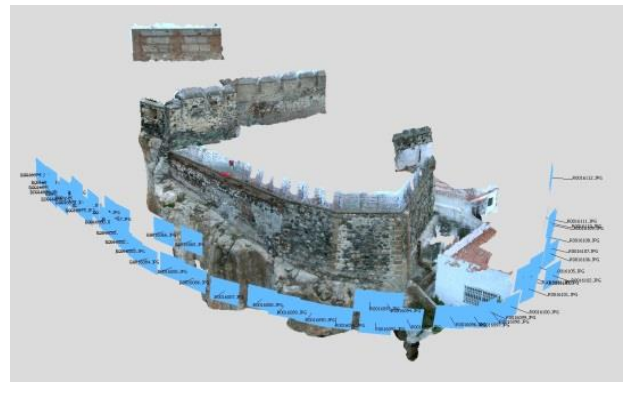

Fig. 3- Modelo 3D generado con software de escaneado fotogramétrico.

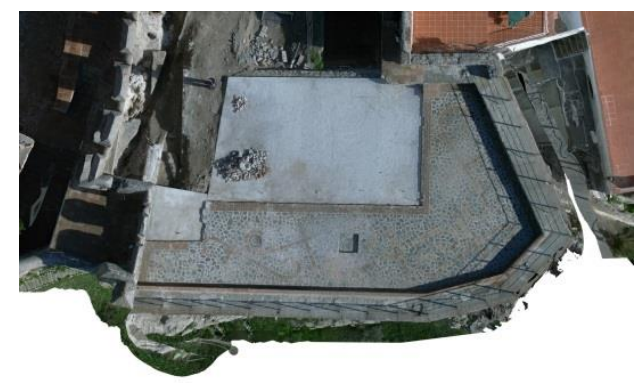

Fig. 4- Ortofoto de la planta del baluarte meridional del Castillo de Salobreña.

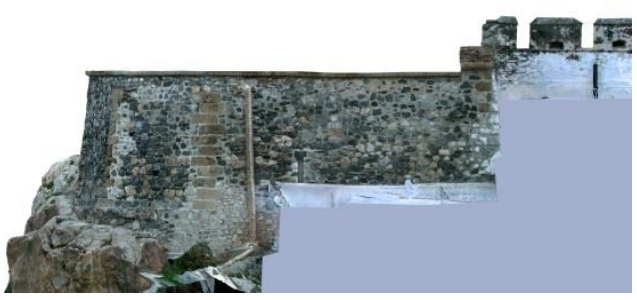

Fig. 5- Ortofoto del alzado oriental del baluarte meridional del Castillo de Salobreña.

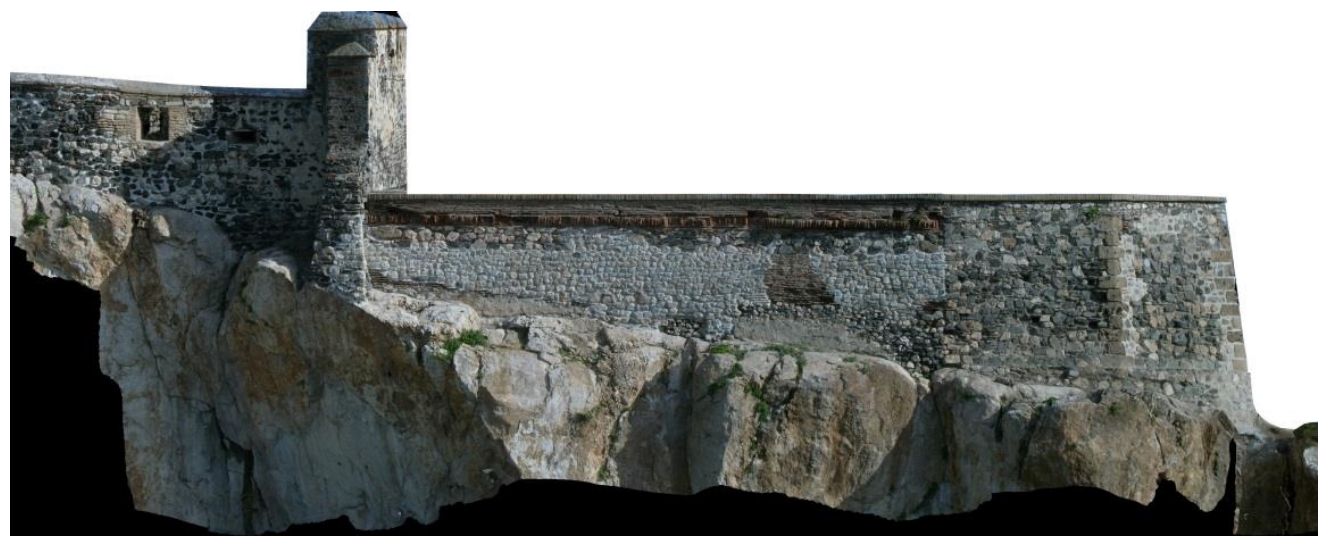

Fig. 6- Ortofoto del alzado que da al acantilado del baluarte meridional del Castillo de Salobreña.

\section{Restauración de zonas en que no es posible la colocación de andamios}

Con frecuencia los problemas antes enunciados que afectan a la documentación, llevan acarreados otros similares en lo que atañe a la intervención de consolidación y restauración. Partes de la fortaleza situadas en zonas inaccesibles suelen plantear dificultades para trabajar sobre ellas al ser muy difícil, o en todo caso muy costoso, el montar andamios o medios auxiliares adecuados para poder realizar los trabajos. Es bastante habitual que la mera instalación de estos medios auxiliares supere con mucho el coste neto de las reparaciones a efectuar que pueden reducirse muchas veces a realizar un rejuntado de las fábricas o en todo caso a rellenar algún hueco 
que se haya producido por desprendimiento de piedras o ladrillos.

En el castillo de Salobreña se presentaba este problema en toda la zona asentada en los bordes del acantilado, cuya altura hacía muy difícil y costoso disponer de andamios para actuar en ella. Por este motivo se decidió recurrir al sistema denominado "trabajo de altura" realizado por operarios especializados con experiencia en ascensión de paredes rocosas en montañas que realizan su trabajo colgados de las paredes por medio de dispositivos de escalada con los consiguientes sistemas de seguridad. Para ello, aparte de disponer los adecuados procedimientos de anclaje y fijación de las cuerdas de las que van colgados, se requiere montar un pequeño dispositivo, generalmente una simple trócola, para hacerles llegar los materiales.

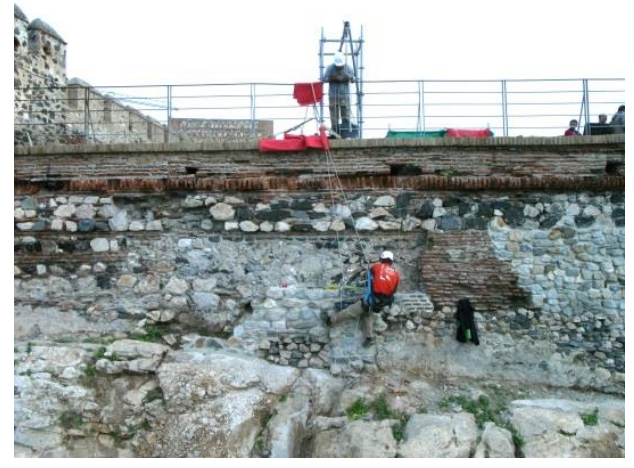

Fig. 7- Operario con arnés y equipo de escalada reparando la fábrica en la muralla del castillo de Salobreña.

Aparte de las labores de rejuntado de las fábricas en toda esta zona, en el frente del baluarte fue preciso desmontar un forro de mampostería que se encontraba totalmente desprendido del resto del muro, y rehacerlo, operación que fue efectuada enteramente con este procedimiento, con un notable ahorro de coste económico e igualmente de tiempo.

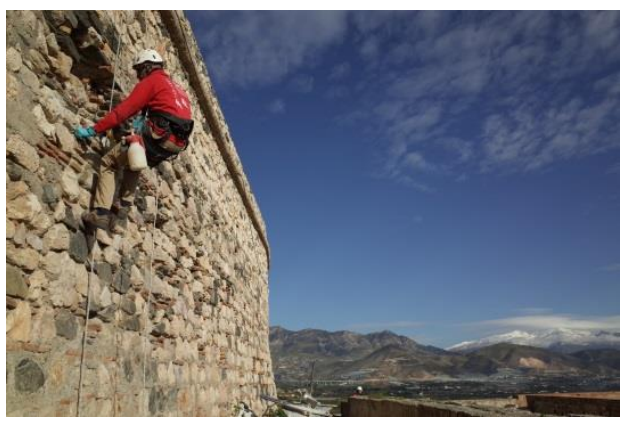

Fig. 8.- Reparación del frente del baluarte sur del castillo de salobreña mediante el método de "trabajo de altura". Vista tomada con un multicóptero provisto de cámara fotográfica.

\section{Conclusiones}

Los problemas que surgen a menudo en labores de documentación y restauración de fortalezas y castillos, a causa de su emplazamiento e inaccesibilidad pueden hoy resolverse acudiendo a sistemas innovadores disponibles cada vez más ampliamente, lo que evita que estas operaciones queden sin hacerse o se hagan con un costo desproporcionado. El uso de ingenios voladores dirigidos por radiocontrol y dotados de cámaras fotográficas y el recurso a los sistemas denominados "trabajos de altura" pueden solventar estas dificultades.

\section{Referencias}

Peinado Checa, Z., Fernández Morales, A., Agustín Hernández, L., (2014) “Combinación de fotogrametría terrestre y aérea de bajo coste: el levantamiento tridimensional de la iglesia de San Miguel de Ágreda (Soria)", Virtual Archaeology Review, vol. 5, n 10, pp. 51-58. 